\title{
V643 Orionis: A detached, evolved, post-mass-exchange eclipsing binary ${ }^{\star}$
}

\author{
J. Andersen ${ }^{1,2}$, G. Torres ${ }^{3}$, and J. V. Clausen ${ }^{1, \star \star}$ \\ 1 The Niels Bohr Institute, University of Copenhagen, Blegdamsvej 17, 2100 Copenhagen, Denmark \\ e-mail: ja@nbi.ku.dk \\ 2 Stellar Astrophysics Centre, Department of Physics and Astronomy, Aarhus University, 8000 Aarhus C, Denmark \\ 3 Center for Astrophysics | Harvard \& Smithsonian, Cambridge, MA 02138, USA \\ e-mail: gtorres@cfa.harvard.edu
}

Received 31 December 2018 / Accepted 8 March 2019

\begin{abstract}
Context. One of the greatest uncertainties in modelling the mass-exchange phases during the evolution of a binary system is the quantity of mass and angular momentum that has been lost from the system. To constrain this problem, a favourable, evolved, and detached real binary system is valuable as an example of the end result of this process.

Aims. We study the 52-day post-mass-exchange eclipsing binary V643 Ori from complete uvby light curves and high-resolution spectra. V643 Ori is double-lined and shows total primary eclipses. The orbit is accurately circular and the rotation of the two stars is synchronised with the orbit, but the photometry from a single year (1993) shows signs of weak spot activity (0.02 mag) around the primary eclipse.

Results. We determined accurate masses of 3.3 and $1.9 M_{\odot}$ from the spectroscopic orbit and solved the four light curves to determine radii of 16 and $21 R_{\odot}$, using the Wilson-Devinney photometric code. The rotational velocities from the cross-correlation profiles agree well with those computed from the known radii and orbital parameters. All observable parameters are thus very precisely determined, but the masses and radii of V643 Ori are incompatible with undisturbed post-main-sequence evolution.

Conclusions. We have attempted to simulate the past evolutionary history of V643 Ori under both conservative and non-conservative Case B mass transfer scenarios. In the non-conservative case we varied the amounts of mass and angular momentum loss needed to arrive at the present masses in a circular 52-day orbit, keeping the two stars detached and synchronised as now observed, but without following the evolution of other stellar properties in any detail. Multiple possible solutions were found. Further attempts were made using both the BSE formalism and the binary MESA code in order to track stellar evolution more closely, and make use of the measured radii and temperatures as important additional constraints. Those efforts yielded no satisfactory solutions, possibly due to limitations in handling mass transfer in evolved stars such as these. We remain hopeful that future theoreticians can more fully model the system under realistic conditions.
\end{abstract}

Key words. stars: evolution - stars: interiors - stars: rotation - binaries: eclipsing

\section{Introduction}

The 52.4-day G-K binary star V643 Orionis (HDE 294651) was first studied in depth by Imbert (1987), who observed a doublelined spectroscopic orbit with the photoelectric radial-velocity scanner CORAVEL (Baranne et al. 1979) on the $1 \mathrm{~m}$ Swiss telescope at Observatoire de Haute Provence and the $1.5 \mathrm{~m}$ Danish telescope at ESO, La Silla, Chile. Imbert got all the main properties of the present system essentially right and found that both components are detached, synchronously rotating giants, but failed to notice the significant fact that the primary and secondary radii were in the opposite ratio of the masses and luminosities.

Since then, we have obtained complete, well-covered $u v b y$ light curves and even more precise radial-velocity curves, and thoroughly analysed them to get a complete, detailed picture of the system in its present state. This paper documents our obser-

* Table 3 and the spectra (in FITS format) are only available at the CDS via anonymous ftp to cdsarc.u-strasbg.fr (130.79.128.5) or via http://cdsarc.u-strasbg.fr/viz-bin/ qcat?]/A+A/624/A88

$\star \star$ Deceased June 5, 2011. vations, analysis, and results on the system. In this way, we hoped to gain some insight into the physical processes that shape the evolution of interacting binaries in general, notably about the processes of mass and angular momentum loss that determine the final outcome. Our original goal, to provide a unique description of the evolution of V643 Ori from the initial configuration to the final state, turned out not to be quite possible, but we eventually came close.

\section{Spectroscopy}

\subsection{Observations and associated results}

Spectroscopic observations of V643 Ori were made at the Center for Astrophysics, Harvard \& Smithsonian (CfA) between October of 1987 and February of 1989 with an echelle spectrograph $(R \approx 35000)$ attached to the $1.5 \mathrm{~m}$ Wyeth reflector at the Oak Ridge Observatory (now closed) in the town of Harvard, Massachusetts. A single order was recorded with an intensified photon-counting Reticon detector providing $45 \AA$ of wavelength coverage centred on the $\mathrm{Mg} 1 \mathrm{~b}$ triplet near $5187 \AA$. A total of 44 usable spectra were collected with signal-to-noise ratios ranging 
from 13 to 24 per resolution element of $8.5 \mathrm{~km} \mathrm{~s}^{-1}$. The velocity zero point was monitored by means of nightly sky exposures taken at dusk and dawn (see Latham 1992).

Radial velocities (RVs) for the two components were measured as described by Torres et al. (2015) with the twodimensional cross-correlation algorithm TODCOR (Zucker \& Mazeh 1994), using templates taken from a pre-computed library of synthetic spectra based on model atmospheres by R. L. Kurucz for solar metallicity (see Nordström et al. 1994; Latham et al. 2002). The optimal template for each star was found by running grids of cross-correlations over a wide range of effective temperatures $\left(T_{\text {eff }}\right)$ and rotational broadenings $(v \sin i)$ following Torres et al. (2002), and selecting the combination giving the highest cross-correlation value averaged over all observations, with weights set by the strength of each exposure. In this way we estimated temperatures of $5210 \pm 150 \mathrm{~K}$ and $4520 \pm 150 \mathrm{~K}$ for the primary (the more massive star) and secondary, and projected rotational velocities of $18 \pm 2 \mathrm{~km} \mathrm{~s}^{-1}$ and $23 \pm 3 \mathrm{~km} \mathrm{~s}^{-1}$, respectively. In view of the masses, age, and previous history of V643 Ori, we assumed a standard Population I chemical composition throughout.

We adopted surface gravity $(\log g$ ) values of 2.5 and 2.0, close to our final determinations from the analysis below. Small adjustments of typically under $1 \mathrm{~km} \mathrm{~s}^{-1}$ were applied to the velocities to correct for systematic errors caused by lines shifting in and out of the narrow spectral window as a function of orbital phase (see Latham et al. 1996). The resulting radial velocities in the heliocentric frame including all corrections are listed in Table 1, along with their uncertainties. The light ratio of the components was determined to be $\ell_{2} / \ell_{1}=0.498 \pm 0.018$ at the mean wavelength of our observations.

In addition to our own measurements, we made use below of the radial-velocity measurements for V643 Ori by Imbert (1987), obtained between 1977 and 1986 with the CORAVEL instrument (Baranne et al. 1979). The projected rotational velocities (Benz $\&$ Mayor 1981, 1984) reported by Imbert (1987) for the primary and secondary star are $17.2 \pm 0.3 \mathrm{~km} \mathrm{~s}^{-1}$ and $21.6 \pm 0.5 \mathrm{~km} \mathrm{~s}^{-1}$, consistent with our results, though significantly more precise.

\subsection{Spectroscopic orbital solution}

Independent spectroscopic orbital fits using our own RVs and those of Imbert (1987) gave results in good agreement with each other. For the CfA measurements we detected a small, but statistically significant systematic offset between the primary and secondary velocities of $-0.65 \pm 0.16 \mathrm{~km} \mathrm{~s}^{-1}$ (primary minus secondary) that may be due to template mismatch, or perhaps the presence of spots on the presumably more active secondary (see below). Neither set of observations indicated any significant eccentricity.

For the final spectroscopic solution we combined the two RV data sets with times of minimum light from our own photometry and from the literature (Table 4 below), which help to improve the ephemeris. We allowed for a zero-point offset between the primary and secondary velocities from CfA, and a similar but independent offset for the CORAVEL velocities. We additionally allowed for a global shift between the CfA and CORAVEL velocities. Separate scale factors for the internal RV errors and for the uncertainties of the times of eclipse were iterated to achieve reduced $\chi^{2}$ values near unity for each component and each type of observation. Once again, tests indicate that the orbit is consistent with being circular.

A weighted least-squares fit to the data gives the elements listed in Table 2. The fit and the observations can be seen in Fig. 1.
Table 1. Heliocentric radial velocities of V643 Ori from CfA.

\begin{tabular}{|c|c|c|c|c|c|}
\hline $\begin{array}{c}\text { HJD } \\
2400000+\end{array}$ & $\begin{array}{r}R V_{1} \\
\left(\mathrm{~km} \mathrm{~s}^{-1}\right)\end{array}$ & $\begin{array}{c}\sigma_{1} \\
\left(\mathrm{~km} \mathrm{~s}^{-1}\right)\end{array}$ & $\begin{array}{r}R V_{2} \\
\left(\mathrm{~km} \mathrm{~s}^{-1}\right)\end{array}$ & $\begin{array}{c}\sigma_{2} \\
\left(\mathrm{~km} \mathrm{~s}^{-1}\right)\end{array}$ & $\begin{array}{c}\text { Orbital } \\
\text { phase }\end{array}$ \\
\hline 47100.9101 & -3.18 & 0.44 & 93.73 & 0.93 & 0.2326 \\
\hline 47461.8790 & 7.88 & 0.45 & 74.14 & 0.95 & 0.1185 \\
\hline 47462.8107 & 5.61 & 0.46 & 79.39 & 0.98 & 0.1362 \\
\hline 47463.7724 & 2.96 & 0.49 & 83.71 & 1.03 & 0.1546 \\
\hline 47463.8096 & 2.61 & 0.43 & 84.22 & 0.92 & 0.1553 \\
\hline 47465.7904 & -0.87 & 0.45 & 90.36 & 0.95 & 0.1931 \\
\hline 47465.8673 & -1.88 & 0.38 & 91.68 & 0.81 & 0.1946 \\
\hline 47469.8568 & -3.21 & 0.36 & 93.35 & 0.76 & 0.2707 \\
\hline 47487.7424 & 55.91 & 0.70 & -6.75 & 1.47 & 0.6118 \\
\hline 47488.6968 & 59.78 & 0.67 & -13.14 & 1.43 & 0.6301 \\
\hline 47489.7456 & 61.94 & 0.63 & -15.93 & 1.33 & 0.6501 \\
\hline 47490.7798 & 64.76 & 0.46 & -20.57 & 0.97 & 0.6698 \\
\hline 47491.7388 & 66.71 & 0.39 & -24.16 & 0.83 & 0.6881 \\
\hline 47491.8272 & 66.32 & 0.41 & -24.17 & 0.88 & 0.6898 \\
\hline 47492.6853 & 68.18 & 0.41 & -24.82 & 0.88 & 0.7061 \\
\hline 47494.7860 & 68.44 & 0.61 & -30.53 & 1.30 & 0.7462 \\
\hline 47496.7754 & 67.29 & 0.59 & -28.54 & 1.26 & 0.7842 \\
\hline 47497.7134 & 67.04 & 0.43 & -25.84 & 0.91 & 0.8021 \\
\hline 47497.7826 & 66.61 & 0.43 & -25.37 & 0.92 & 0.8034 \\
\hline 47512.6629 & 13.19 & 0.57 & 65.99 & 1.20 & 0.0872 \\
\hline 47513.8190 & 9.03 & 0.47 & 72.10 & 1.00 & 0.1093 \\
\hline 47514.8394 & 6.11 & 0.54 & 78.62 & 1.13 & 0.1288 \\
\hline 47515.6919 & 3.50 & 0.42 & 82.34 & 0.88 & 0.1450 \\
\hline 47522.6724 & -3.26 & 0.45 & 94.47 & 0.94 & 0.2782 \\
\hline 47522.7456 & -2.83 & 0.54 & 92.35 & 1.13 & 0.2796 \\
\hline 47526.5811 & 3.89 & 0.42 & 83.62 & 0.90 & 0.3527 \\
\hline 47527.6685 & 6.80 & 0.41 & 77.24 & 0.88 & 0.3735 \\
\hline 47538.6520 & 50.06 & 0.44 & 1.06 & 0.94 & 0.5830 \\
\hline 47544.6200 & 67.01 & 0.39 & -25.99 & 0.83 & 0.6969 \\
\hline 47546.5788 & 68.44 & 0.41 & -28.57 & 0.87 & 0.7342 \\
\hline 47547.5260 & 68.63 & 0.63 & -26.86 & 1.33 & 0.7523 \\
\hline 47548.5882 & 67.61 & 0.43 & -26.69 & 0.92 & 0.7726 \\
\hline 47549.5577 & 67.47 & 0.44 & -27.52 & 0.93 & 0.7910 \\
\hline 47549.6979 & 68.19 & 0.44 & -25.14 & 0.93 & 0.7937 \\
\hline 47550.6414 & 65.92 & 0.37 & -24.40 & 0.79 & 0.8117 \\
\hline 47550.8994 & 65.75 & 0.37 & -23.77 & 0.79 & 0.8166 \\
\hline 47551.6600 & 64.19 & 0.43 & -19.91 & 0.92 & 0.8311 \\
\hline 47554.6145 & 56.40 & 0.46 & -8.93 & 0.97 & 0.8875 \\
\hline 47569.5650 & 0.34 & 0.40 & 89.24 & 0.86 & 0.1727 \\
\hline 47569.6841 & 0.16 & 0.45 & 88.55 & 0.95 & 0.1750 \\
\hline 47570.5943 & -0.74 & 0.41 & 92.20 & 0.86 & 0.1923 \\
\hline 47574.5598 & -4.27 & 0.45 & 95.70 & 0.95 & 0.2680 \\
\hline 47575.5136 & -2.71 & 0.44 & 94.96 & 0.94 & 0.2862 \\
\hline 47576.5013 & -2.83 & 0.44 & 92.21 & 0.93 & 0.3050 \\
\hline
\end{tabular}

Notes. Orbital phases are computed from Eq. (1) below.

\section{Photometry}

\subsection{Observations}

V643 Ori was observed in the uvby photometric system on a total of 119 nights between 1988 and 1994 with the Danish $50 \mathrm{~cm}$ Strömgren Automatic Telescope (SAT) and its six-channel photometer, using a 17" diaphragm. HD 42131, HD 41873, and HD 41143 were selected as comparison stars and were observed alternately with V643 Ori. They were found to be constant to within 4 mmag (rms) throughout the period, and the instrument has also seen extensive use in the same period for other work. 
Table 2. Spectroscopic orbital solution for V643 Ori.

\begin{tabular}{|c|c|}
\hline Parameter & Value \\
\hline \multicolumn{2}{|c|}{ Adjusted elements } \\
\hline$P$ (days) & $52.42136 \pm 0.00014$ \\
\hline$\gamma\left(\mathrm{km} \mathrm{s}^{-1}\right)$ & $+32.557 \pm 0.067$ \\
\hline$K_{1}\left(\mathrm{~km} \mathrm{~s}^{-1}\right)$ & $36.307 \pm 0.073$ \\
\hline$K_{2}\left(\mathrm{~km} \mathrm{~s}^{-1}\right)$ & $61.99 \pm 0.16$ \\
\hline Min I (HJD $2400000+$ ) & $47193.554 \pm 0.016$ \\
\hline$\Delta_{\mathrm{CfA}}\left(\mathrm{km} \mathrm{s}^{-1}\right)$ & $-0.64 \pm 0.16$ \\
\hline$\Delta_{\text {CORAVEL }}\left(\mathrm{km} \mathrm{s}^{-1}\right)$ & $-0.40 \pm 0.51$ \\
\hline$\Delta\left(\mathrm{km} \mathrm{s}^{-1}\right)$ & $-0.84 \pm 0.23$ \\
\hline \multicolumn{2}{|c|}{ Derived quantities } \\
\hline$M_{1} \sin ^{3} i\left(\mathcal{M}_{\odot}^{\mathrm{N}}\right)$ & $3.254 \pm 0.019$ \\
\hline$M_{2} \sin ^{3} i\left(\mathcal{M}_{\odot}^{\mathrm{N}}\right)$ & $1.9056 \pm 0.0090$ \\
\hline$q \equiv M_{2} / M_{1}$ & $0.5857 \pm 0.0019$ \\
\hline$a \sin i\left(\mathcal{R}_{\odot}^{\mathrm{N}}\right)$ & $101.86 \pm 0.18$ \\
\hline $\operatorname{CfA} \sigma_{1}, \sigma_{2}\left(\mathrm{~km} \mathrm{~s}^{-1}\right)$ & $0.44 / 0.95$ \\
\hline CfA $N_{\mathrm{obs}, 1}, N_{\mathrm{obs}, 2}$ & $44 / 44$ \\
\hline CORAVEL $\sigma_{1}, \sigma_{2}\left(\mathrm{~km} \mathrm{~s}^{-1}\right)$ & $1.31 / 2.54$ \\
\hline CORAVEL $N_{\text {obs }, 1}, N_{\text {obs }, 2}$ & $35 / 31$ \\
\hline$N_{\text {Min I }}, N_{\text {Min II }}$ & $19 / 9$ \\
\hline
\end{tabular}

Notes. $\Delta_{\mathrm{CfA}}$ and $\Delta_{\mathrm{CORAVEL}}$ are the primary minus secondary velocity offsets, $\Delta$ is the global CfA minus CORAVEL shift. The minimum masses and semimajor axis are in units of the nominal solar mass and radius (2015 IAU Resolution B3, see Prša et al. 2016).

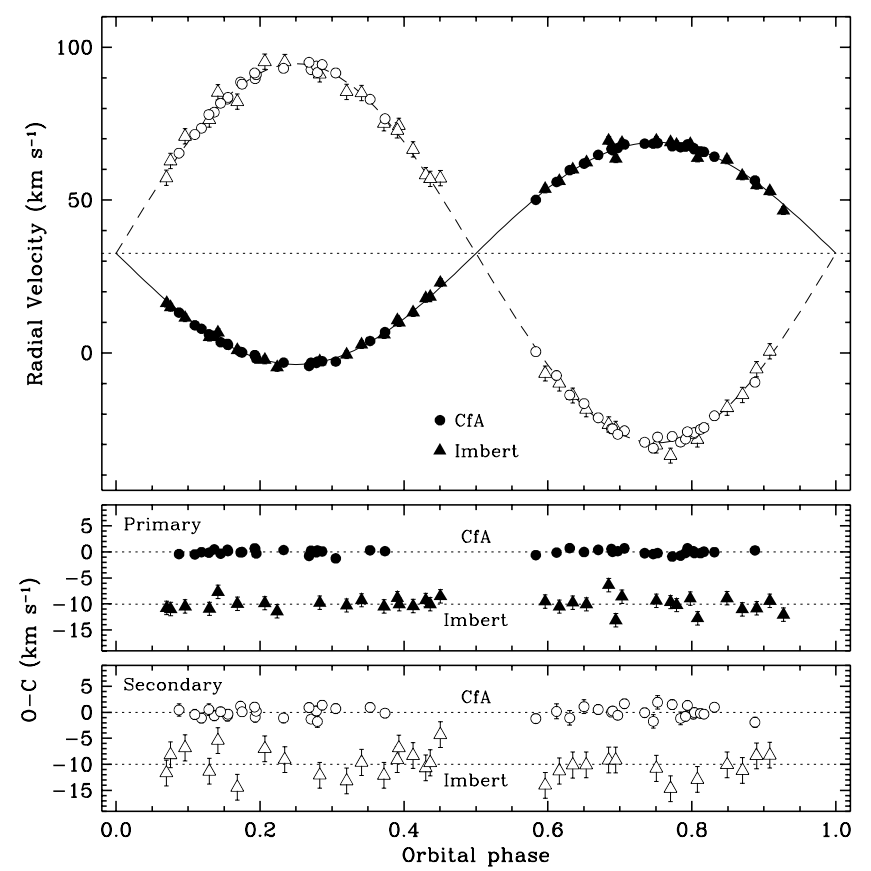

Fig. 1. Spectroscopic orbital solution for V643 Ori. Primary observations are shown with filled symbols; the dotted line in the top panel marks the centre-of-mass velocity of the system. Residuals are shown at the bottom; the Imbert (1987) residuals are shifted for clarity.

Details of the extinction corrections and transformations to the standard system are as described by Olsen (1994).

The light curves contain a total of 559 points in each colour, with typical mean errors for a single differential magnitude of $0.008 \mathrm{mag}(v b y)$ and $0.010-0.015 \mathrm{mag}(u)$; the $y$ observations

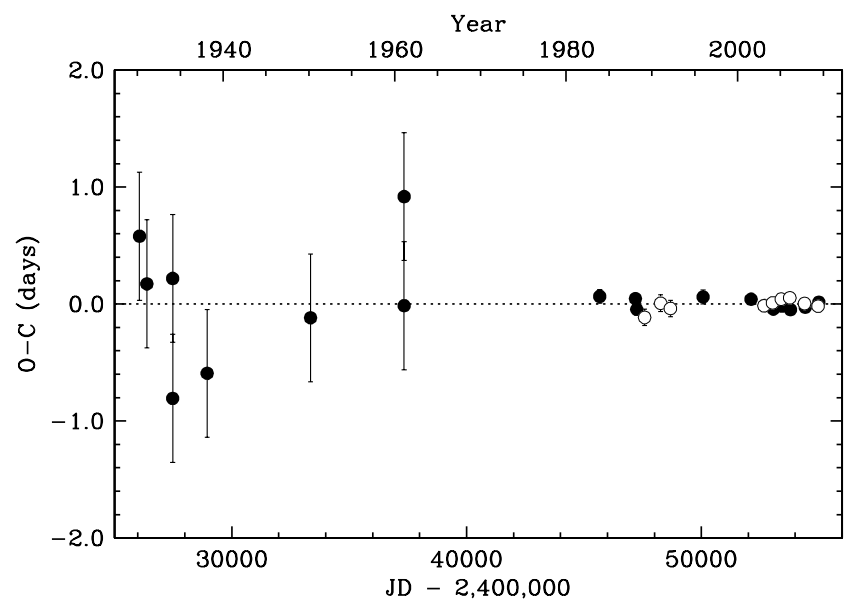

Fig. 2. Times of minimum listed in Table 4 . The period seems to have been constant over 80 years.

from one season (35 observations on seven nights in 1993) fall $\sim 0.02$ mag below the rest (presumably due to slight spot activity) and were simply omitted in the following analysis. All 524 remaining observations are available electronically (only) in Table 3, and are shown graphically in Fig. 3. The duration of the eclipses is about 5.5 days; Min I shows a totality interval of only $\sim 16 \mathrm{~h}$.

Standard $u v b y \beta$ indices of V643 Ori at maximum light were measured relative to a grid of standard stars (see Olsen 1993, 1994, for details), and are $V=9.365 \pm 0.007, b-y=0.812 \pm$ $0.004, m_{1}=0.237 \pm 0.026$, and $c_{1}=0.482 \pm 0.018$ based on 10 measurements, and $\beta=2.591$ (single measurement).

Times of minimum light determined by the Kwee \& van Woerden (1956) method from groups of consecutive nights covering the eclipses are presented in Table 4, along with other such measurements from the literature. No significant period change is detectable, and the final linear ephemeris we derive from a joint analysis with the spectroscopy (Table 2) is:

Min I = HJD $2447193.554+52.42136 E$.

As can be seen in Fig. 2, the period has been constant within the limitations of the observations, contrary to the speculations of Eggleton \& Yakut (2017) about a possible third star in the system. V643 Ori is well detached, and the primary eclipse, when the cooler and larger secondary is in front, has a short interval of totality (see above), so the luminosity of both stars is accurately determined.

\subsection{Light curve solution}

We modelled the four uvby light curves simultaneously using the Wilson-Devinney light curve model and programme (Wilson \& Devinney 1971; Wilson 1979) version $2013^{1}$ in mode 2, called within a Markov chain Monte Carlo (MCMC) framework in order to sample the posterior distributions of the adjusted parameters. The main variables we explored are the surface potentials $\Omega_{1}$ and $\Omega_{2}$, the secondary temperature $T_{\mathrm{eff}, 2}$, the inclination angle $i$, and the out-of-eclipse magnitude $m_{0}$ for each pass band, at phase 0.25 . The primary temperature was held fixed at the spectroscopic value $T_{\text {eff, } 1}=5210 \mathrm{~K}$ (Sect. 2 ).

We also fixed the period and reference epoch of primary eclipse to the values in Eq. (1) and Table 2, but we allowed

1 ftp://ftp.astro.ufl.edu/pub/wilson/lcdc2013/ 


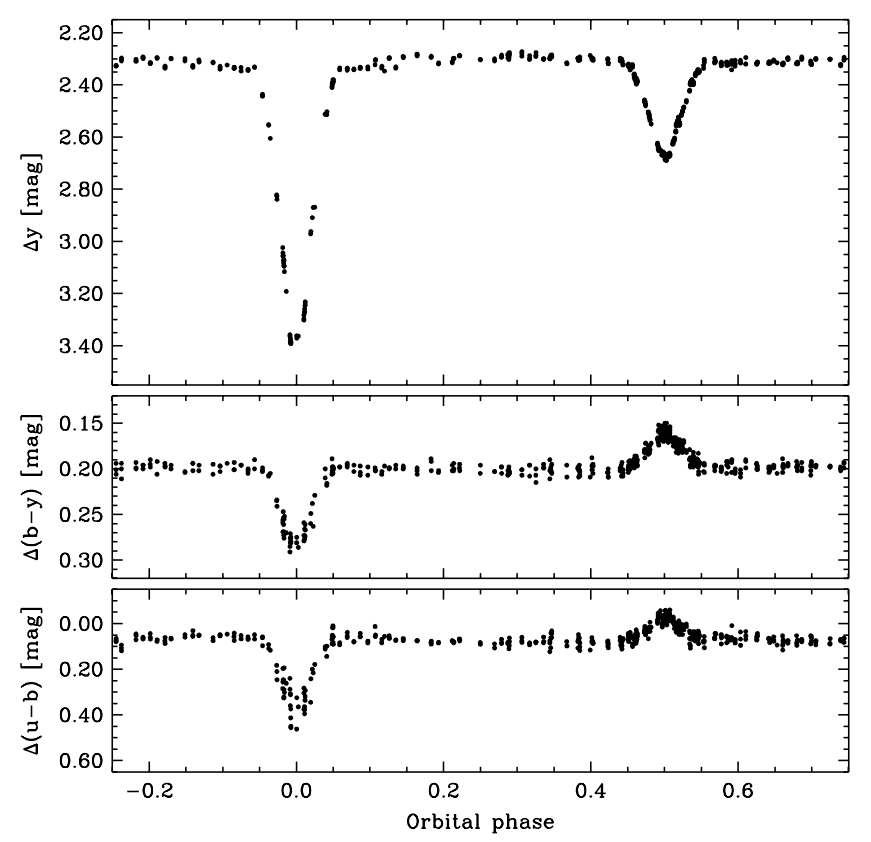

Fig. 3. Differential magnitudes and colours for V643 Ori on the instrumental system.

for minor phase adjustments $\Delta \phi$ separately for each pass band. Scale factors $f_{\sigma}$ for the initial photometric uncertainties in $u v b y$ ( $\sigma=0.02 \mathrm{mag}$ ) were included as free parameters as well, with the addition of appropriate terms to the likelihood function (Gregory 2005). The mass ratio was held at the spectroscopic value of $q=0.5857$, and gravity darkening exponents (0.32) and bolometric albedos $(0.5)$ set to values appropriate for stars with convective envelopes. To model the local emission from the two stars we used the model atmosphere option in the code. Limb darkening coefficients $u_{1}$ and $u_{2}$ were taken from the tables of Claret \& Bloemen (2011) for the linear law (ATLAS models), as a more complicated treatment is not warranted given the visible distortions in the light curves caused by spots.

Our method of solution used the emcee ${ }^{2}$ code of Foreman-Mackey et al. (2013), which is a Python implementation of the affine-invariant MCMC ensemble sampler proposed by Goodman \& Weare (2010). Convergence was judged by visual inspection of the chains, along with the requirement of a Gelman-Rubin statistic smaller than 1.05 for each parameter (Gelman \& Rubin 1992).

Initial runs resulted in an obvious systematic and wavelength-dependent pattern to the residuals during the secondary eclipse, likely related to the presence of spots. We find that this could be corrected by allowing the limb darkening coefficient of that star to be a free parameter. The primary limb darkening coefficients were held fixed, as varying them did not improve the residuals any further. Other tests that included third light gave values of $L_{3}$ not significantly different from zero that also brought no improvement, so our final runs were carried out with $L_{3}=0$ in all bands. The correlations among some of the key variables may be appreciated in the diagram of Fig. 4, generated from our final solution.

Time-correlated noise in the light curves as a result of the presence of spots causes the formal uncertainties of the adjusted parameters to be underestimated. To account for this, we carried out a residual permutation exercise in which we simulated

\footnotetext{
2 https://dfm.io/emcee/current/
}

Table 4. Times of minimum light for V643 Ori.

\begin{tabular}{|c|c|c|c|c|c|}
\hline $\begin{array}{c}\text { HJD } \\
(2400000+)\end{array}$ & $\begin{array}{c}\sigma \\
\text { (days) } \\
\end{array}$ & Eclipse & Type & Year & Source \\
\hline 26068.325 & 0.530 & 1 & pg & 1930.2487 & 1 \\
\hline 26382.446 & 0.530 & 1 & pg & 1931.1087 & 1 \\
\hline 27482.315 & 0.530 & 1 & pg & 1934.1200 & 1 \\
\hline 27483.341 & 0.530 & 1 & pg & 1934.1228 & 1 \\
\hline 28950.328 & 0.530 & 1 & pg & 1938.1392 & 1 \\
\hline 33354.197 & 0.530 & 1 & pg & 1950.1963 & 2 \\
\hline 37338.324 & 0.530 & 1 & pg & 1961.1042 & 2 \\
\hline 37339.257 & 0.530 & 1 & pg & 1961.1068 & 2 \\
\hline 45673.400 & 0.057 & 1 & $\mathrm{v}$ & 1983.9244 & 2 \\
\hline 47193.600 & 0.046 & 1 & pe & 1988.0865 & 3 \\
\hline 47245.930 & 0.046 & 1 & pe & 1988.2298 & 3 \\
\hline 47586.600 & 0.074 & 2 & pe & 1989.1625 & 3 \\
\hline 48268.200 & 0.074 & 2 & pe & 1991.0286 & 3 \\
\hline 48687.525 & 0.074 & 2 & pe & 1992.1767 & 3 \\
\hline 50076.79 & 0.057 & 1 & $\mathrm{v}$ & 1995.9803 & 4 \\
\hline 52121.20335 & 0.031 & 1 & pe & 2001.5776 & 5 \\
\hline 52671.57191 & 0.031 & 2 & pe & 2003.0844 & 5 \\
\hline 52697.78540 & 0.031 & 1 & pe & 2003.1562 & 5 \\
\hline 53038.54694 & 0.031 & 2 & pe & 2004.0891 & 5 \\
\hline 53064.70369 & 0.031 & 1 & pe & 2004.1607 & 5 \\
\hline 53405.52748 & 0.031 & 2 & pe & 2005.0938 & 5 \\
\hline 53431.67806 & 0.031 & 1 & pe & 2005.1654 & 5 \\
\hline 53772.48842 & 0.031 & 2 & pe & 2006.0985 & 5 \\
\hline 53798.59786 & 0.031 & 1 & pe & 2006.1700 & 5 \\
\hline 54401.49642 & 0.031 & 2 & pe & 2007.8207 & 5 \\
\hline 54427.67544 & 0.031 & 1 & pe & 2007.8923 & 5 \\
\hline 54978.10783 & 0.031 & 2 & pe & 2009.3993 & 5 \\
\hline 55004.35238 & 0.031 & 1 & pe & 2009.4712 & 5 \\
\hline
\end{tabular}

Notes. "Eclipse" is 1 for primary minimum, 2 for secondary. "Type" indicates photographic (pg), visual (v), or photoelectric (pe). Sources are: (1) Mauder (1960); (2) Online Bundesdeutsche Arbeitsgemeinschaft für Veränderliche Sterne (BAV) database (http: //var2.astro.cz/ocgate/index.php?lang=en); (3) this paper; (4) Hübscher et al. (1997); (5) Zasche et al. (2011).

$u v b y$ data sets that were based on the residuals from our adopted solution below. We shifted the residuals by a random number of time steps and added them back into the adopted model light curves at each time of observation. We generated 50 such artificial data sets and submitted each one to a new MCMC analysis. Each of these used randomly perturbed values for all previously fixed quantities, including the primary limb darkening coefficients, the bolometric albedos, and the gravity darkening exponents (all assumed to be normally distributed around their adopted values, with $\sigma=0.1$ ).

We also applied Gaussian perturbations to the mass ratio and primary effective temperature by amounts consistent with their measured uncertainties. We calculated the scatter of the distributions for each parameter, and combined those values in quadrature with the internal errors from our nominal solution to arrive at the final uncertainties. Table 5 gives the final light curve elements and adopted uncertainties.

\section{Discussion}

\subsection{Absolute dimensions}

The preceding sections have described the analysis of our spectroscopic and photometric observations of V643 Ori. Here we 


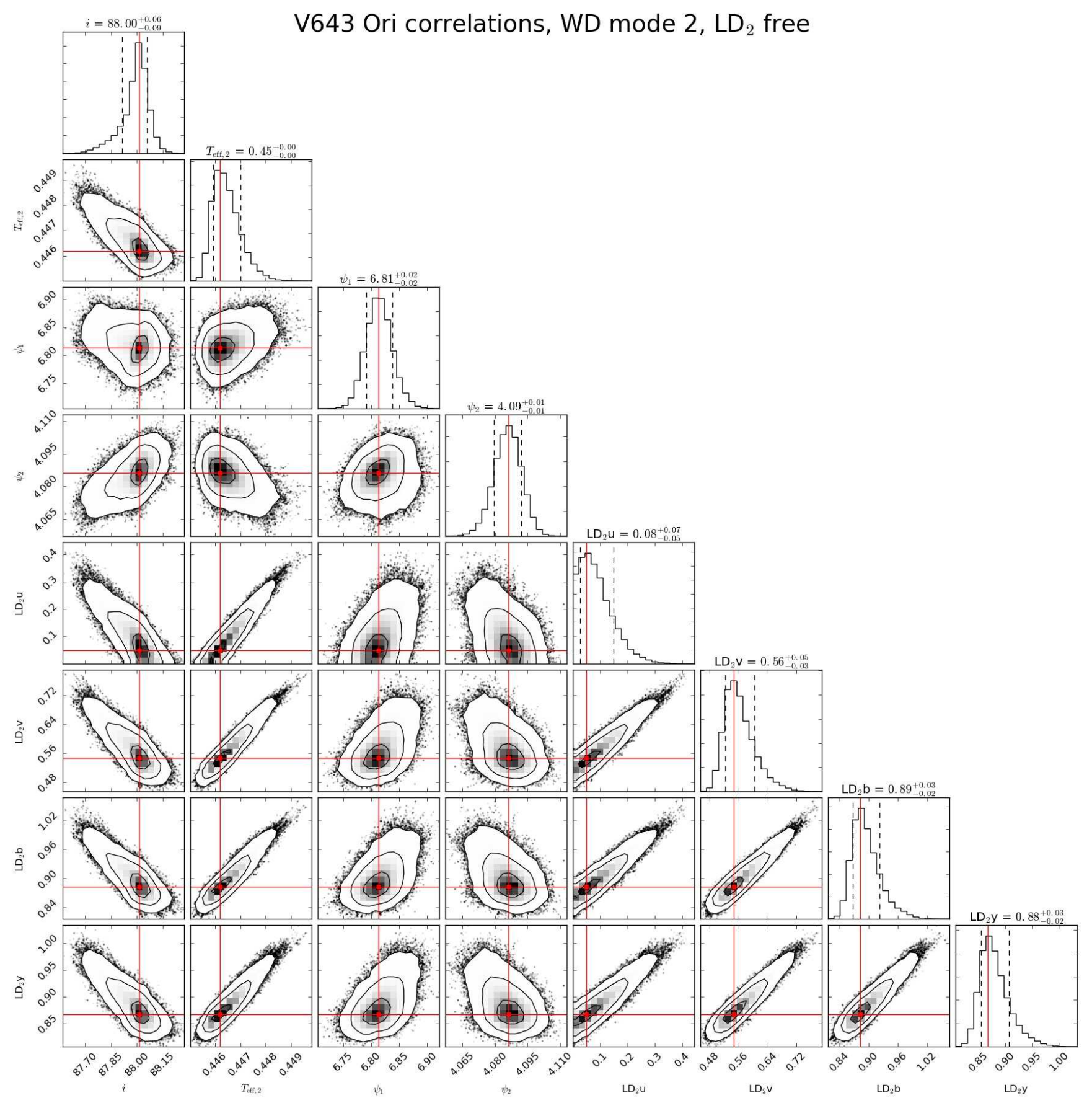

Fig. 4. "Corner plot" (source code available at https://github.com/dfm/corner.py, Foreman-Mackey 2016) for V643 Ori, illustrating the correlations among the main fit parameters of our solution. Contour levels correspond to 1,2 , and $3 \sigma$, and the histograms on the diagonal represent the posterior distribution for each parameter, with the mode and internal 68\% confidence levels indicated. More realistic errors are discussed in the text.

combine the computed results of Tables 2 and 5 into the coherent, comprehensive and accurate picture of the physical properties of the V643 Ori system in its present state, which is summarised in Table 6.

The absolute masses and all orbital parameters are determined to about $0.5 \%$ real accuracy and the radii to $1.5 \%$, or better. Both components are near-spherical and relatively far separated from their Roche lobes (Table 5) as expected, since the orbit has been circularised; V643 Ori is a well-detached system. The measured component rotations are those expected for the observed radii and the period (and inclination) of the circular orbit.
The radiative properties of V643 Ori are less precisely determined. A reddening estimate of $E(B-V)=0.298 \pm 0.096$ was taken from the tri-dimensional map of the local interstellar medium of Capitanio et al. (2017) and used to estimate the luminosities of the stars, adopting a distance to V643 Ori of $1270 \mathrm{pc}$ from the Gaia/DR2 catalogue. The independent distance estimate we derive, $840 \pm 130 \mathrm{pc}$, is somewhat smaller than the trigonometric value, possibly due to small errors in our (primary) effective temperature, or in the reddening. Thus, the present state of V643 Ori is very precisely determined, thanks both to the very favourable configuration of V643 Ori itself and to our careful 
Table 5. Adopted light curve solution for V643 Ori.

\begin{tabular}{|c|c|c|c|c|}
\hline Parameter & \multicolumn{2}{|c|}{ Primary } & \multicolumn{2}{|c|}{ Secondary } \\
\hline$\Omega$ & \multicolumn{2}{|c|}{$6.813_{-0.094}^{+0.095}$} & \multicolumn{2}{|c|}{$4.085_{-0.023}^{+0.023}$} \\
\hline$r_{\text {pole }}$ & \multicolumn{2}{|c|}{$0.1604 \pm 0.0024$} & \multicolumn{2}{|c|}{$0.2021 \pm 0.0015$} \\
\hline$r_{\text {point }}$ & \multicolumn{2}{|c|}{$0.1616 \pm 0.0025$} & \multicolumn{2}{|c|}{$0.2101 \pm 0.0018$} \\
\hline$r_{\text {side }}$ & \multicolumn{2}{|c|}{$0.1609 \pm 0.0024$} & \multicolumn{2}{|c|}{$0.2044 \pm 0.0016$} \\
\hline$r_{\text {back }}$ & \multicolumn{2}{|c|}{$0.1615 \pm 0.0025$} & \multicolumn{2}{|c|}{$0.2086 \pm 0.0017$} \\
\hline$r_{\mathrm{vol}}$ & \multicolumn{2}{|c|}{$0.1610 \pm 0.0024$} & \multicolumn{2}{|c|}{$0.2052 \pm 0.0016$} \\
\hline$r_{\text {Roche }}$ & \multicolumn{2}{|c|}{0.429} & \multicolumn{2}{|c|}{0.330} \\
\hline$R_{2} / R_{1}$ & \multicolumn{4}{|c|}{$1.275 \pm 0.018$} \\
\hline$i(\operatorname{deg})$ & \multicolumn{4}{|c|}{$88.02^{+0.34}$} \\
\hline$T_{\mathrm{eff}, 1}(\mathrm{~K})$ & \multicolumn{4}{|c|}{5210 (fixed) } \\
\hline$T_{\mathrm{eff}, 2}(\mathrm{~K})$ & \multicolumn{4}{|c|}{$4462_{-95}^{+96}$} \\
\hline$\Delta T_{\mathrm{eff}}(\mathrm{K})$ & \multicolumn{4}{|c|}{$748 \pm 51$} \\
\hline Parameter & $u$ & $v$ & $b$ & $y$ \\
\hline$u_{1}$ (fixed) & 0.939 & 0.922 & 0.795 & 0.674 \\
\hline$u_{2}$ & $0.05_{-0.05}^{+0.21}$ & $0.54_{-0.12}^{+0.13}$ & $0.88_{-010}^{+0.11}$ & $0.87^{+0.12}$ \\
\hline$\ell_{2} / \ell_{1}$ & $0.370 \pm 0.010$ & $0.445 \pm 0.009$ & $0.546 \pm 0.010$ & $0.616 \pm 0.012$ \\
\hline$\Delta \phi$ & $-0.00045_{-0.00017}^{+0.00017}$ & $-0.00067_{-0.00014}^{+0.00015}$ & $-0.00061_{-0.00015}^{+0.0016}$ & $-0.00055_{-0.00016}^{+0.00016}$ \\
\hline$m_{0}(\mathrm{mag})$ & $2.5768_{-0.0034}^{+0.0034}$ & $2.5315_{-0.0031}^{+0.000014}$ & $2.4992^{+0.00033}$ & $2.3027^{+0.00025}$ \\
\hline & $1.297_{-0.043}^{+0.0524}$ & $0.756_{-0.039}^{+0.042}$ & $0.672_{-0.036}^{+0.039^{2}}$ & $0.671_{-0.041}^{+0.042}$ \\
\hline$\sigma(\mathrm{mag})$ & 0.0260 & 0.0151 & 0.0135 & 0.0134 \\
\hline
\end{tabular}

Table 6. Physical properties of V643 Ori.

\begin{tabular}{lcc}
\hline \multicolumn{1}{c}{ Parameter } & Primary & Secondary \\
\hline$M\left(\mathcal{M}_{\odot}^{\mathrm{N}}\right)$ & $3.260 \pm 0.019$ & $1.9090 \pm 0.0091$ \\
$R\left(\mathcal{R}_{\odot}^{\mathrm{N}}\right)$ & $16.41 \pm 0.25$ & $20.91 \pm 0.17$ \\
$a\left(\mathcal{R}_{\odot}^{\mathrm{N}}\right)$ & $101.92 \pm 0.18$ \\
$\log g(\mathrm{dex})$ & $2.521 \pm 0.013$ & $2.0782 \pm 0.0072$ \\
$T_{\text {eff }}(\mathrm{K})$ & $5210 \pm 150$ & $4460 \pm 180$ \\
$\log L / L_{\odot}$ & $2.252 \pm 0.052$ & $2.194 \pm 0.070$ \\
$M_{\text {bol }}(\mathrm{mag})$ & $-0.90 \pm 0.13$ & $-0.75 \pm 0.17$ \\
$B C_{V}(\mathrm{mag})$ & $-0.22 \pm 0.11$ & $-0.65 \pm 0.18$ \\
$M_{V}(\mathrm{mag})$ & $-0.68 \pm 0.21$ & $-0.10 \pm 0.33$ \\
$\left.v_{\text {sync }} \sin i(\mathrm{~km} \mathrm{~s})^{-1}\right)^{a}$ & $15.83 \pm 0.24$ & $20.27 \pm 0.26$ \\
$\left.v \sin i(\mathrm{~km} \mathrm{~s})^{-1}\right)^{b}$ & $17.2 \pm 0.3$ & $21.6 \pm 0.5$ \\
$E(B-V)(\mathrm{mag})$ & $0.298 \pm 0.093$ \\
$A_{V}(\mathrm{mag})$ & $0.92 \pm 0.29$ \\
Dist. modulus $(\mathrm{mag})$ & \multicolumn{2}{c}{$9.62 \pm 0.34$} \\
Distance $(\mathrm{pc})$ & $840 \pm 130$ \\
$\pi($ mas $)$ & \multicolumn{2}{c}{$1.19 \pm 0.19$} \\
$\pi_{\text {Gaia } / \text { DR2 }}(\mathrm{mas})$ & $0.786 \pm 0.048$ \\
\hline
\end{tabular}

Notes. Masses, radii, and semi-major axis $a$ are expressed in units of the nominal solar mass and radius $\left(\mathcal{M}_{\odot}^{\mathrm{N}}, \mathcal{R}_{\odot}^{\mathrm{N}}\right)$ as recommended by 2015 IAU Resolution B3 (see Prša et al. 2016), and the adopted solar temperature is $5772 \mathrm{~K}$ (2015 IAU Resolution B2). Bolometric corrections are from the work of Flower (1996), with conservative uncertainties of $0.1 \mathrm{mag}$ added in quadrature, and the bolometric magnitude adopted for the Sun appropriate for this $B C_{V}$ scale is $M_{\text {bol }}^{\odot}=4.732$ (see Torres 2010). See text for the source of the reddening. For the apparent visual magnitude of V643 Ori out of eclipse we used $V=9.365 \pm 0.020$ (Sect. 3.1, with a more conservative error). ${ }^{(a)}$ Synchronous projected rotational velocity assuming spin-orbit alignment. ${ }^{(b)}$ Measured projected rotational velocity (Imbert 1987).

analysis to obtain a consistent picture of the system, with few or no loose ends.

\subsection{Evolution of V643 Ori}

Any attempt to model the evolutionary history of V643 Ori must begin by considering its initial configuration and assigning initial masses to the two stars, and a separation between them or (equivalently) the orbital period of the binary. The task is then to match the present configuration of V643 Ori with (i) a single age, (ii) a 52-day circular orbit, and (iii) two evolved, detached, synchronised components, with a physically realistic model for the evolution and mass exchange processes of the stars in V643 Ori. The quantities of mass and angular momentum transferred between the stars, and from the system, may be varied as necessary.

The initial primary star must obviously have been the present secondary, since it is the more highly evolved star, that is, it must have had a mass above $\sim 2.6 M_{\odot}$, which is half the mass of the present system. If mass is conserved, the present secondary star must have had an initial mass substantially lower than this, since it has received some mass from the primary. Now it has become a $\sim 3.3 M_{\odot}$ evolved star, most likely a clump giant in the core He-burning phase - the longest-lived post-main sequence phase (both stars are detached clump giants).

The simplest possible model, though arguably not the most realistic, is one in which both mass and angular momentum are perfectly conserved during mass transfer, and the orbit remains circular. Under those conditions the initial and final masses and orbital periods are related by

$\frac{P_{\text {final }}}{P_{\text {init }}}=\left(\frac{M_{1, \text { init }} M_{2, \text { init }}}{M_{1, \text { final }} M_{2, \text { final }}}\right)^{3}$

(see, e.g. Postnov \& Yungelson 2014). With the final state known, and the initial secondary mass given by $M_{2 \text {,init }}=M_{\text {tot }}-$ $M_{1, \text { init }}$, any choice for the initial orbital period immediately determines the initial primary mass. We illustrate this in Fig. 6.

One possible scenario has the V643 Ori system starting out with the present orbital period of 52 days, and with the initial masses reversed from what we measure. The size of the Roche 


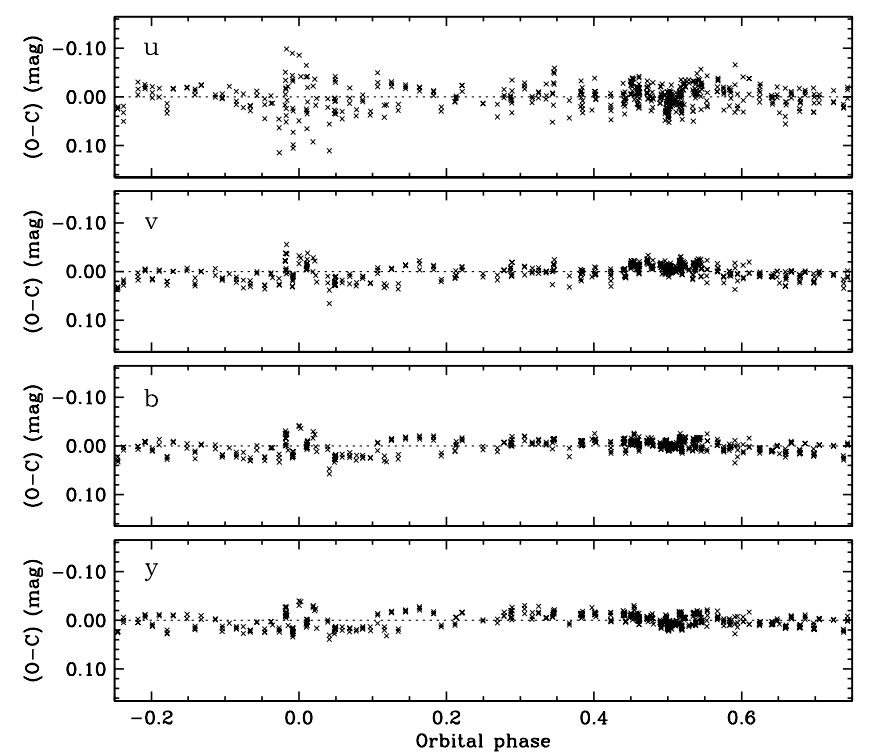

Fig. 5. Light curve residuals in uvby from our final model for V643 Ori.

lobe of the original $3.3 M_{\odot}$ donor would be roughly $34 R_{\odot}$. A solar-metallicity evolutionary track calculated with the MESA code (Paxton et al. 2011, 2013, 2015) shows that a normal star of that mass would reach this size near the tip of the giant branch (first ascent), at an age of about $290 \mathrm{Myr}$, triggering "Case B" mass transfer by Roche lobe overflow (RLOF). Conservation of angular momentum dictates that the orbit must shrink as mass flows from the donor to the less massive gainer until the masses become equal.

With the equation above we calculated that at this point the orbital period reaches a minimum of about 42 days, implying a separation near $90 R_{\odot}$. The $\sim 33 R_{\odot}$ Roche lobes of the two stars are thus well separated, consistent with the notion of conservation of mass. As the transfer of material in this idealised scenario continues, the orbit widens again, and mass exchange ceases when the donor retreats as a core helium burning (CHB or "clump") giant inside its $\sim 44 R_{\odot}$ Roche lobe at the present 52-day period.

By then the former secondary has accreted about $1.4 M_{\odot}$ worth of Population I material, and ends up appearing as a fairly typical $3.3 M_{\odot}$ clump giant. Comparison of its measured properties with those predicted from a MESA evolutionary track shows it to have the right luminosity for a clump giant, while being slightly smaller and hotter than expected. On the other hand, the original primary star, now less massive, but also a CHB giant with a $\sim 0.6 M_{\odot}$ He core, is over-luminous, large, and cool for its mass compared to a normal clump giant, but still fitting comfortably inside its Roche lobe.

While matching many of the present properties of the system, the configuration explored above, with an initial orbital period that is the same as the current one, is of course completely arbitrary and many other choices of initial configuration are possible, according to Fig. 6, all leading to the same final masses and period.

A somewhat more realistic scenario would account for the possibility of mass and angular momentum loss from the system. This may occur, for example, if a fraction $\beta$ of the mass that reaches the vicinity of the gainer is then ejected as a fast, isotropic wind with the specific angular momentum of the gainer. Under these conditions the relationship between the initial and

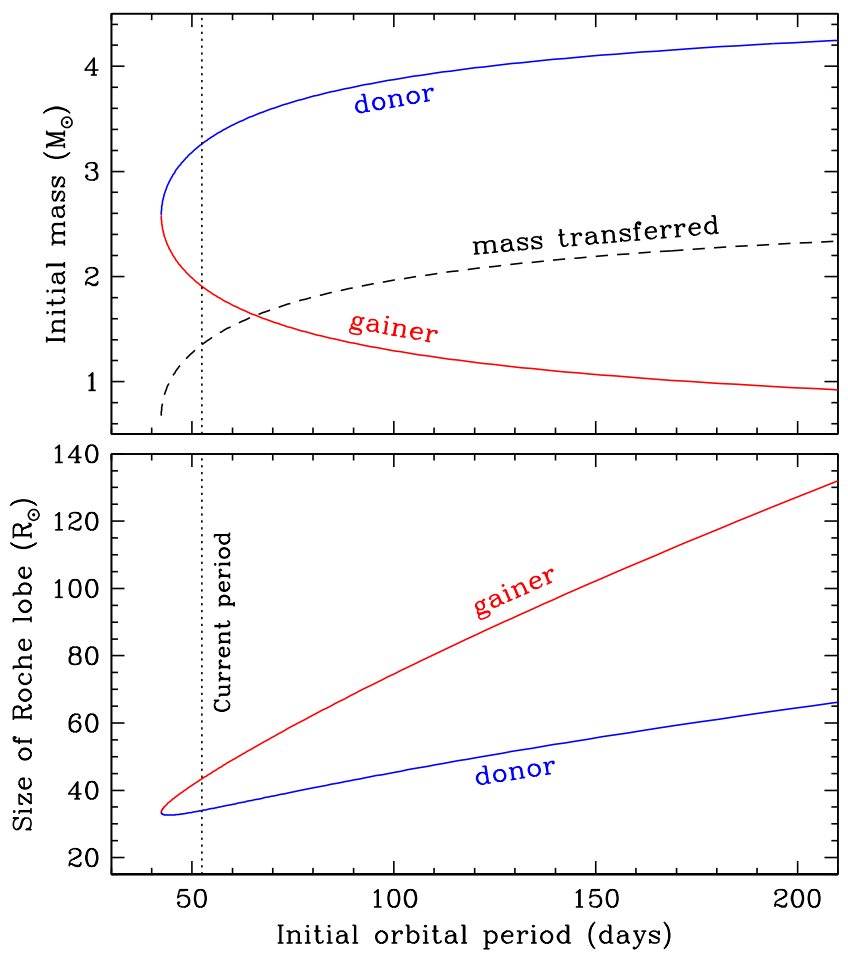

Fig. 6. Initial donor and gainer masses (top) required to match the present masses and orbital period of V643 Ori if total mass and angular momentum are perfectly conserved, as a function of the chosen initial period. The amount of mass transferred is also indicated. Bottom panel: size (radius) of the limiting Roche lobe of the donor (and gainer) at the chosen initial orbital period. Mass transfer by Roche lobe overflow begins when the donor reaches this size during its normal evolution.

final periods becomes

$\frac{P_{\text {final }}}{P_{\text {init }}}=\left(\frac{q_{\text {init }}}{q_{\text {final }}}\right)^{3}\left(\frac{1+q_{\text {init }}}{1+q_{\text {final }}}\right)^{2}\left(\frac{1+(1-\beta) q_{\text {final }}}{1+(1-\beta) q_{\text {inital }}}\right)^{\frac{8-5 \beta}{1-\beta}}$

(see, e.g. Tauris 1996; Soberman et al. 1997), in which the mass ratio is defined as $q \equiv M_{1} / M_{2}$ and star 1 is the donor. The orbit is assumed to remain circular throughout.

For a given initial donor mass, Fig. 7 shows the initial gainer mass and initial orbital period that are required in order to reach the present masses and period after the end of RLOF. This is given as a function of the fraction $\beta$ of mass transferred that is lost from the system in the manner described above.

Other properties of the system resulting from this nonconservative scenario may also be seen in the figure, including the initial radius of the donor's Roche lobe, and the age of the star when it evolves to reach that size, and mass transfer begins. When $\beta=0$, Eq. (3) reduces to Eq. (2) and the results are exactly the same as in the strictly conservative scenario considered earlier. The non-conservative scenario has one more free parameter than the conservative case, and as before, there are many possible sets of initial conditions that lead to the current configuration.

The Soberman et al. (1997) formalism is general enough that other mechanisms of mass and angular momentum loss may be explored as well. One is a spherically symmetric outflow from the donor star in the form of a fast, magnetised wind, carrying with it the specific angular momentum of the donornot unlikely in view of the observed spot(s) presumably on the present secondary (cooler star). Another is mass loss to a circumbinary accretion ring. As an example, the first of these mechanisms is illustrated in Fig. 7 by the dotted lines, calculated for 

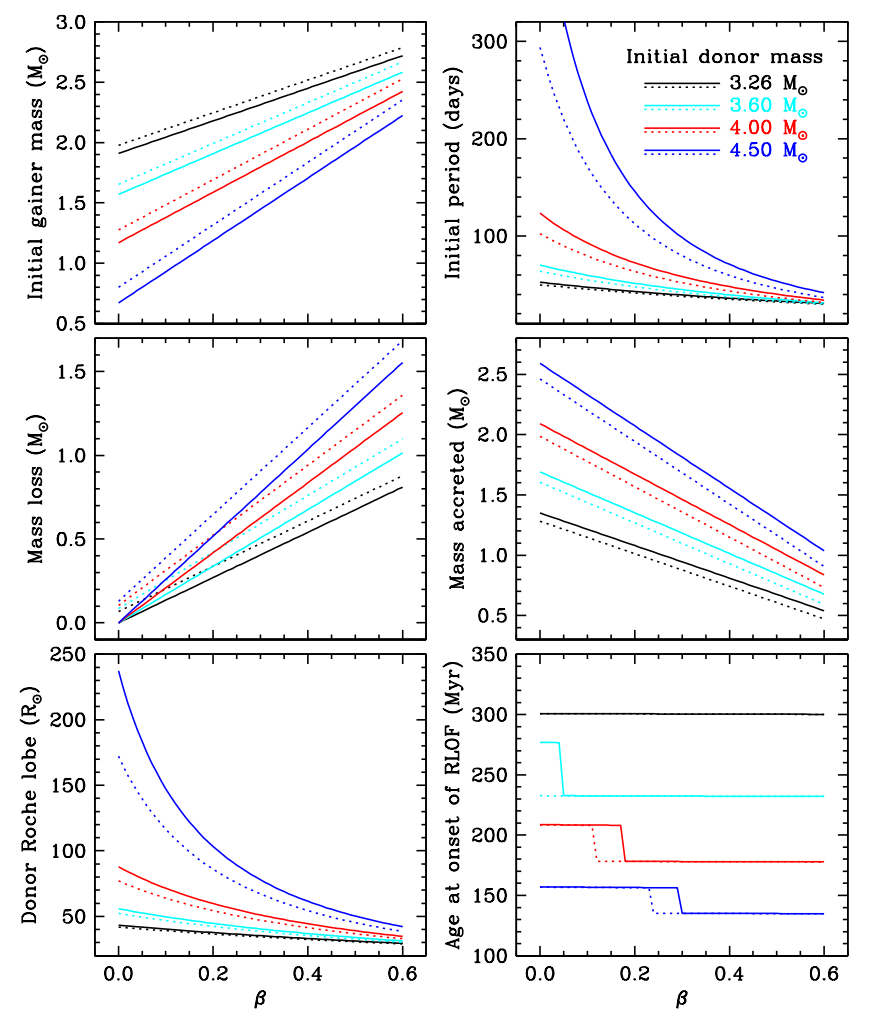

Fig. 7. Initial conditions and other properties of the V643 Ori system under non-conservative mass transfer, calculated with the formalism of Soberman et al. (1997). Results are plotted for four initial donor masses (labelled), as a function of the fraction $\beta$ of the mass transferred from the donor that is ejected from the system in the vicinity of the gainer. Middle panels: amount of mass lost, and amount of material that is actually accreted by the gainer. Bottom panels: size of the donor's Roche lobe at the initial period (left), and age of the system (right) when the donor triggers mass transfer as it reaches that size, according to MESA models for normal solar-metallicity stars. The discontinuities in the age sequences as $\beta$ increases are the result of the initial donor switching from attaining the critical (Roche-lobe) radius during the asymptotic giant branch phase (larger sizes) to reaching it on the first ascent of the giant branch (smaller radii, hence younger ages). Dotted lines in all panels show how the results change when an additional $5 \%$ of the mass transferred is lost to the system via an isotropic wind from the donor star (see text).

a loss of 5\% of the transferred mass (parameter $\alpha$ in the notation of Soberman et al. 1997) through an isotropic wind. The effect is significant, especially at the higher initial donor masses.

Unfortunately, these increasingly complex scenarios add more free parameters, but bring us no closer to a unique solution for V643 Ori. They give us no detailed information about what transpires during mass transfer, which will surely result in very different stellar structures and final sizes and temperatures for the two stars (both of which are well determined) for any given choice of initial conditions.

Furthermore, these scenarios may still be too simplistic; more detailed modelling would be desirable to account for other forms of mass and angular momentum loss from the system that may be at work, as well as for the evolution of the initial orbital eccentricity and rotation rates due to tides (since we know that the present system is circular and synchronised), and even for a possible common-envelope phase. This should allow important additional constraints such as the measured radii and temperatures of the components to narrow the range of possible solutions, ideally down to a single one.
At our request, attempts in that direction were carried out by J. Andrews using the BSE code (Hurley et al. 2002) and also by A. Dotter with the more sophisticated MESA binary code (Paxton et al. 2015). Both computer programmes make trade-offs and have their own limitations: BSE is able to follow the binary system through the common-envelope phase in an approximate way, but makes many simplifications in dealing with evolution; MESA treats stellar evolution more rigorously, but cannot handle the common-envelope phase. As a result, neither experiment succeeded in identifying a plausible initial state leading to the current properties of the system.

With BSE, a wide range of initial properties leads to the system entering a common-envelope phase, from which it emerges with masses and/or an orbital period very different from what we measure. The attempts to model the system with MESA, on the other hand, seem to fail because it is not designed for systems in which mass transfer begins when the donor is already a giant, as is likely the case for V643 Ori. In the end, therefore, these efforts were less satisfactory than the simpler calculations described above.

\section{Conclusion}

Our original goal of properly modelling in detail the evolution of V643 Ori, taking into account the exchange of mass and angular momentum and their loss from the stars and from the system itself, thus eluded us, even though the end product - the present system - was so precisely specified. At the present time, theoretical models (especially for convective giant stars) seem inadequate to treat these processes in sufficient detail to allow the use of all observational constraints at the same time, and in that way to identify a unique solution.

In the meantime, we have at least explored the variation of the masses and orbital period under conservative and nonconservative scenarios, though without following the evolution of other stellar properties in any detail. When full models allowing this finally do become available, we shall have at least one carefully observed system and its properties to look for.

Acknowledgements. Service observers J. Caruso, R. Davis, R. Stefanik, and J. Zajac helped with the spectroscopic observations, and several SAT observers included V643 Ori in their programmes; we thank them all. Jeff Andrews and Aaron Dotter spent considerable time in trying to model the evolution of V643 Ori with MESA and other suites of evolutionary codes, and P. Maxted helped early on with the determination of the stellar properties. JVC participated fully in the observations and their reduction, so he clearly merits posthumous coauthorship, but he obviously bears no responsibility for the analysis and final text of the paper. We thank the careful anonymous referee for providing very helpful comments on the original manuscript, which led to extensive modifications. GT acknowledges partial support from the NSF through grant AST-1509375, and the use of the Danish telescopes on La Silla was supported by the then Danish Natural Science Research Council (now the Independent Research Fund Denmark | Natural Sciences). This research has made use of the SIMBAD database, operated at the CDS, Strasbourg, France, and of NASA's Astrophysics Data System Abstract Service.

\section{References}

Baranne, A., Mayor, M., \& Poncet, J. L. 1979, Vistas in Astron., 23, 279 Benz, W., \& Mayor, M. 1981, A\&A, 93, 235

Benz, W., \& Mayor, M. 1984, A\&A, 138, 183

Capitanio, L., Lallement, R., Vergely, J. L., Elyajouri, M., \& Monreal-Ibero, A. 2017, A\&A, 606, A65

Claret, A., \& Bloemen, S. 2011, A\&A, 529, A75

Eggleton, P. P., \& Yakut, K. 2017, MNRAS, 468, 3533

Flower, P. J. 1996, ApJ, 469, 355

Foreman-Mackey, D. 2016, J. Open Sour. Soft., 24 
Foreman-Mackey, D., Hogg, D. W., Lang, D., \& Goodman, J. 2013, PASP, 125, 306

Gelman, A., \& Rubin, D. B. 1992, Statistical Science, 7, 457

Goodman, J., \& Weare, J. 2010, Appl. Math. Comput. Sci., 5, 65

Gregory, P. C. 2005, ApJ, 631, 1198

Hübscher, J., Agerer, F., Hassforther, B., \& Wunder, E. 1997, BAV Mitt., 101, 2

Hurley, J. A., Tout, C. A., \& Pols, O. R. 2002, MNRAS, 329, 897

Imbert, M. 1987, A\&AS, 71, 69

Kwee, K. K., \& van Woerden, H. 1956, Bull. Astron. Inst. Netherlands., 12, 327

Latham, D. W. 1992, in IAU Coll., 135, Complementary Approaches to Double and Multiple Star Research, eds. H. A. McAlister, \& W. I. Hartkopf (San Francisco: ASP), ASP Conf. Ser., 32, 110

Latham, D. W., Nordström, B., Andersen, J., et al. 1996, A\&A, 314, 864

Latham, D. W., Stefanik, R. P., Torres, G., et al. 2002, AJ, 124, 1144

Mauder, H. 1960, Veröff Reimeis-Sternw. Bamberg, band V, No. 7

Nordström, B., Latham, D. W., Morse, J. A., et al. 1994, A\&A, 287, 338

Olsen, E. H. 1993, A\&A, 102, 89
Olsen, E. H. 1994, A\&A, 106, 257

Paxton, B., Bildsten, L., Dotter, A., et al. 2011, ApJS, 192, 3

Paxton, B., Cantiello, M., Arras, P., et al. 2013, ApJS, 208, 4

Paxton, B., Marchant, P., Schwab, J., et al. 2015, ApJS, 220, 15

Postnov, K. A., \& Yungelson, L. R. 2014, Liv. Rev. Relativ., 17, 3

Prša, A., Harmanec, P., Torres, G., et al. 2016, AJ, 152, 41

Soberman, G. E., Phinney, E. S., \& van den Heuvel, E. P. J. 1997, A\&A, 327, 620

Tauris, T. M. 1996, A\&A, 315, 453

Torres, G. 2010, AJ, 140, 1158

Torres, G., Neuhäuser, R., \& Guenther, E. W. 2002, AJ, 123, 1701

Torres, G., Sandberg Lacy, C. H., Pavlovski, K., Fekel, F. C., \& Muterspaugh, M. W. 2015, AJ, 150, 154

Wilson, R. E. 1979, ApJ, 234, 1054

Wilson, R. E., \& Devinney, E. J. 1971, ApJ, 166, 605

Zasche, P., Uhlar, R., Kucáková, H., \& Svoboda, P. 2011, IBVS, 6007

Zucker, S., \& Mazeh, T. 1994, ApJ, 420, 806 